\begin{tabular}{|c|c|}
\hline 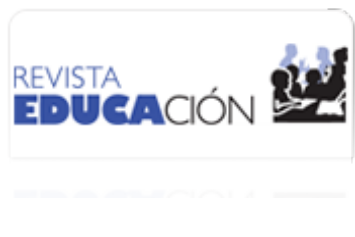 & $\begin{array}{l}\text { Revista Educación } \\
\text { ISSN: 0379-7082 } \\
\text { ISSN: 2215-2644 } \\
\text { revedu@gmail.com } \\
\text { Universidad de Costa Rica } \\
\text { Costa Rica }\end{array}$ \\
\hline
\end{tabular}

\title{
Suficiencia y equidad de la infraestructura escolar en el Perú: un análisis por departamentos y regiones naturales $^{[1]}$
}

\author{
Sánchez Alcalde, Luis Amado \\ Suficiencia y equidad de la infraestructura escolar en el Perú: un análisis por departamentos y regiones \\ naturales ${ }^{[1]}$ \\ Revista Educación, vol. 44, núm. 2, 2020 \\ Universidad de Costa Rica, Costa Rica \\ Disponible en: http://www.redalyc.org/articulo.oa?id=44062184028 \\ DOI: https://doi.org/10.15517/revedu.v44i2.39190
}

Esta obra está bajo una Licencia Creative Commons Atribución-NoComercial-SinDerivar 3.0 Internacional. 


\title{
Suficiencia y equidad de la infraestructura escolar en el Perú: un análisis por departamentos y regiones naturales ${ }^{[1]}$
}

\author{
Adequacy and Equity of School Infrastructure in Peru: An Analysis Based on Departments and Natural Regions
}

Luis Amado Sánchez Alcalde

Universidad Autónoma Metropolitana, México

luis.sanchezalcalde@gmail.com

(D) http://orcid.org/0000-0001-7501-4421

\author{
DOI: https://doi.org/10.15517/revedu.v44i2.39190 \\ Redalyc: http://www.redalyc.org/articulo.oa?id=44062184028
}

Recepción: 14 Noviembre 2019

Aprobación: 28 Mayo 2020

\section{RESUMEN:}

La literatura señala que la infraestructura de las escuelas forma parte de los insumos que intervienen en la producción de los resultados educativos. La evidencia en América Latina y el Caribe señala que esta tiene un efecto positivo sobre el rendimiento del estudiantado; sin embargo, se observan problemas de suficiencia y equidad. El objetivo del presente trabajo es describir la infraestructura escolar en el Perú bajo los criterios de suficiencia y equidad señalados por la evidencia empírica en la región. Para ello se construye un indicador sintético a partir de la información estadística del MINEDU, con el cual se pudo observar que la suficiencia de infraestructura escolar atraviesa problemas en los departamentos que se ubican en la región natural Sierra, existiendo resultados mejores en la Costa y en la zona metropolitana. Los resultados tienen una tendencia similar para los años 2005 y 2017, lo que conllevaría a concluir que la equidad en la suficiencia de la infraestructura de las escuelas del Perú no ha cambiado sustancialmente en más de diez años.

Palabras Clave: Infraestructura escolar, Suficiencia, Equidad, Índice sintético, Perú.

\section{Aвstract:}

Previous studies have emphasized that school facilities play a key role in advancing educational results. Latin American and the Caribbean have proven that this aspect has a positive impact on student achievement. Nonetheless, issues regarding the adequacy and equity of infrastructure and facilities were observed. The objective of this study is to describe school facilities in Peru based on a criteria of adequacy and equality according to empirical evidence from the region. A synthetic indicator was elaborated using statistics from the Peruvian Ministry of Education (MINEDU). It was observed that adequacy of school facilities currently faces constraints, particularly, in the Departments of the Highland Natural Region. More favorable results were observed along the Coastal and Metropolitan Area. All results reveal a similar trend from the years 2005 to 2017, which reflects that equity as pertains to the quality of school facilities in Pero has not undergone any substantial changes for more than ten years.

KEYwords: Sufficiency, Equality, School Infrastructure, Facilities, Synthetic Index, Peru.

\section{INTRODUCCIÓN}

La literatura indica que uno de los insumos que influye en la producción de conocimientos y habilidades del estudiantado es la infraestructura escolar. Actualmente existen estudios para América Latina y el Caribe que respaldan lo anterior; sin embargo, la evidencia también muestra la presencia de problemas de suficiencia y equidad. De acuerdo con algunos autores, la suficiencia se relaciona con los requerimientos mínimos de infraestructura que aseguran el aprendizaje, así como la funcionalidad de los ambientes y el mobiliario escolar. En el caso de la equidad esta se relaciona con la justicia e igualdad del entorno escolar. En general, la equidad en la infraestructura escolar tiene que ver con el hecho de que todo el alumnado acceda a recursos similares sin importar condición alguna, como por ejemplo el área geográfica. De esto se desprende que en un sistema educativo no basta solo con la infraestructura per se, sino que esta debe ser de suficiente y accesible a todo el estudiantado. 
En el Perú, se ha propuesto el Plan Nacional de Infraestructura Escolar al 2025 el cual tiene como objetivo mejorar la condición, capacidad, gestión y sostenibilidad de la infraestructura educativa pública para lograr una educación de calidad. Uno de los objetivos específicos del plan es atender de manera óptima la demanda aún no cubierta, es decir, construir infraestructura accesible a cada estudiante. En este sentido, la presente investigación plantea la siguiente pregunta: En el Perú, ¿la suficiencia de la infraestructura escolar se concentra en una determinada región natural? Considerando que en el Perú existen tres regiones naturales: costa, sierra y selva, la hipótesis es que una infraestructura escolar considerada como suficiente se encuentra concentrada en los departamentos de la región natural costa. Dado esto, el objetivo fue describir la suficiencia y equidad de la infraestructura escolar a través de un índice sintético usando los datos que ofrece el Censo Escolar del Ministerio de Educación del Perú - MINEDU y el método de Análisis de Componentes Principales (ACP).

El trabajo se divide de la siguiente forma: Primero se define la infraestructura como insumo del rendimiento escolar; luego se revisan los resultados de los estudios en América Latina y el Caribe. Posteriormente, se presentan las características de los departamentos del Perú y algunos resultados de la educación básica. En la sección metodológica se muestra cuáles fueron los criterios usados para la construcción del índice sintético. Finalmente, se brindan los resultados y conclusiones preliminares.

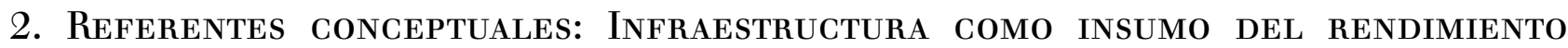 ESCOLAR}

La economía de la educación se basa en la Función de Producción de la Educación - FPE para analizar qué insumos producen los resultados educativos (Carnoy, 2006). Uno de estos insumos es la infraestructura escolar, la cual representa un componente esencial en el proceso educativo (Duarte, Jaureguiberry y Racimo, 2017). En el modelo de Cameron, Daga y Outhred (2018) se propone que los principales insumos en el proceso de la producción de la educación son: (i) el gasto público, (ii) gasto privado y (iii) el capital humano y social de los padres y madres del estudiantado. Estos a la vez producen insumos intermedios como la calidad de docentes y las facilidades de la escuela, los que van a producir finalmente el rendimiento escolar. Otro modelo clasifica a los insumos de la FPE con base en la demanda y oferta educativa (Beltrán y Seinfeld, 2013). En cuanto a la demanda, se tiene al capital humano y social del entorno de cada estudiante (siguiendo lo propuesto por Cameron et al., 2018); mientras que por el lado de la oferta se tienen factores como: el hardware educativo (Harbinson y Hanushek, 1992) representado por la infraestructura de las aulas, el acceso a servicios de agua, energía eléctrica, entre otros aspectos edilicios; el software educativo representado por la gestión educativa, autonomía escolar, entre otros; y finalmente la calidad de las y los docentes como su nivel educativo alcanzado, años de experiencia, entre otros (Beltrán y Seinfeld, 2013).

Por otro lado, la calidad educativa no solo se basa en los resultados sino también en los insumos (Jain y Prasad, 2018). En este sentido, el diseño del llamado hardware educativo debe ser de calidad para lograr la máxima efectividad (Barrett, Treves, Shmis, Ambasz, y Ustinova, 2019), y debe adecuarse a las necesidades del estudiantado. Siguiendo a Quesada (2019), “el concepto de espacio escolar debe visualizarse como un espacio estéticamente agradable donde se motive al estudiante a vivenciar un proceso de enseñanza y aprendizaje exitoso" (p.3). Cada nivel educativo requiere de cierta infraestructura, siendo esta distinta para el Inicial (Hille, 2012), Primario (O’Donnell, 2012) y Secundario (Fornari, 2012). Literatura reciente indica que la conceptualización del diseño de las escuelas se encuentra en un periodo de cambio dada la importancia que representa la infraestructura en el aprendizaje; al respecto Duarte et al. (2017) señalan que

los estudios contemporáneos sobre infraestructura escolar y calidad de la educación se orientan cada vez más a entender cómo los espacios físicos de aprendizaje producen condiciones y mediaciones que facilitan tanto los resultados académicos como el bienestar de los estudiantes. Enfatizan más la calidad de los espacios que la mera existencia de los mismos (p.8). 
De esta forma, el concepto tradicional de escuela está cambiando por nociones más amplias como ambientes de aprendizaje, espacios físicos de aprendizaje o espacios de aprendizaje (Duarte et al., 2017). Otro aspecto importante sobre la calidad de la infraestructura y la relación con el aprendizaje es su mantenimiento, Quesada (2019) señala que no solo se debe mantener el edificio escolar sino también el mobiliario, el cual debe ser suficiente para lograr la calidad educativa.

Además de la calidad, la infraestructura debe ser accesible a todo el estudiantado en un sentido de equidad (Barrett et al., 2019). La equidad educativa tiene relación con conceptos como igualdad y justicia (Cameron et al. 2018) y podría definirse como las libertades fundamentales que adquieren los individuos a lo largo del sistema educativo (Formichella, 2011). Para el Center for Public Education - CPE, la equidad es lograda cuando el estudiantado recibe los recursos necesarios para graduarse y tener éxito después de la escuela secundaria; mientras que la igualdad se logra cuando todo el alumnado es tratado igual y tiene acceso a recursos similares. En cuanto a la infraestructura escolar, la equidad significa que todo el estudiantado sin importar su género, habilidades especiales, ubicación geográfica y condición socioeconómica tengan acceso a recursos suficientes (Barrett et al., 2019; Duarte et al., 2017).

En conclusión, la infraestructura escolar es un insumo del complejo proceso educativo cuya importancia radica en que debe ser de calidad y disponible para todo el estudiantado, de esta manera se lograrán los resultados deseados. En la Figura 1 se muestra la secuencia de los modelos de FPE mencionados comenzando por los insumos ya descritos que finalmente producirán los resultados escolares.

\section{Infraestructura escolar en AmÉrica LATINA}

La información sobre el estado de la infraestructura de las escuelas forma parte del conjunto de estadísticas educativas que los países han recolectado y organizado en las últimas décadas. La evidencia empírica del efecto positivo que tiene la infraestructura sobre el rendimiento del estudiantado es amplia. Por ejemplo, con base en una revisión de más de 100 investigaciones, Barrett et al. (2019) han propuesto cinco dimensiones que se relacionan con los entornos de aprendizaje:

- Accesibilidad a la infraestructura de la escuela.

- Buena construcción de las escuelas (salud y seguridad).

- Espacios óptimos para aprendizaje.

- Sinergia con la pedagogia y la comunidad.

- Efectiva implementación del proyecto escolar.

La mayoría de estudios que analizan estas dimensiones se encuentra en los países desarrollados. Recientemente la disponibilidad de datos para América Latina ha permitido observar lo que sucede en la región, de esta manera los resultados encontrados ya forman parte de los reportes sobre el tema. Duarte, Gargiulo y Moreno (2011) y Duarte et al. (2017) muestran la situación de la infraestructura escolar y la relación que existe entre esta y el rendimiento de alumnos de educación básica (tercer y sexto grado del nivel primario) en países de América Latina y el Caribe. Se escogieron estas investigaciones no solo por su relevancia académica sino también por la metodología usada, pues los autores proponen un criterio de calidad de la infraestructura escolar a partir de la suficiencia de un conjunto de componentes considerados como indispensables para lograr el aprendizaje. Estas investigaciones usaron la base de datos del Segundo y Tercer Estudio Regional Comparativo y Explicativo de la calidad educativa (SERCE y TERCE) ${ }^{[2]}$ respectivamente, los cuales representan esfuerzos por tener información estadística consistente para la región.

Duarte et al. (2011) describe que la infraestructura y el acceso a los servicios básicos de electricidad, agua, desagüe y teléfono son altamente deficientes en las escuelas de América Latina y el Caribe, observándose diferencias entre las escuelas urbanas y rurales, públicas y privadas. En términos desagregados, los países de 
Centroamérica (exceptuando Costa Rica) y República Dominicana presentan los mayores problemas en infraestructura escolar; por el contrario, Chile, Argentina y Uruguay tienen mejores condiciones; mientras que México, Brasil y Colombia se encuentran cerca del promedio. En el caso de la energía eléctrica, Nicaragua, Perú, Panamá y Guatemala presentan carencias importantes. El acceso al servicio de agua potable también presenta problemas en países centroamericanos (a excepción de Costa Rica) y en Colombia, Perú, Ecuador y Paraguay. Por el lado del servicio de desagüe, los casos más agudos son los de Nicaragua, Paraguay, Guatemala, Perú, Panamá, República Dominicana y El Salvador; asimismo, el número de baños para alumnos es crítico para Centroamérica, México, Perú, Paraguay, Ecuador y Colombia.

Los autores construyeron siete índices de infraestructura escolar con base en los datos de infraestructura del SERCE, y utilizando el método de Análisis de Componentes Principales (ACP):

1. Índice de áreas deportivas: Campo o cancha deportiva.

2. Índice de áreas de usos múltiples: Gimnasio y Auditorio.

3. Índice de área de oficinas: Oficinas para el director, oficinas adicionales y sala de reuniones para profesores.

4. Índice de áreas Académicas/pedagógicas: Laboratorio de ciencias, sala de computación, biblioteca, sala de artes o música.

5. Índice áreas de salud: Enfermería y servicio psicopedagógico.

6. Índice de servicios: Luz eléctrica y Teléfono.

7. Índice de agua y saneamiento: Agua potable, desagüe, baños en cantidad suficiente.

Entre los resultados de esta metodología, se observó por ejemplo que para el índice de Áreas Académicas/ Pedagógicas, Chile, Argentina y Cuba se posicionan por arriba del promedio de la región; mientras que Nicaragua, Paraguay República Dominicana, están por debajo. Asimismo, los índices se utilizaron como variable explicativa del rendimiento escolar, usando modelos de regresión multinivel, cuyos resultados indicaron que las bibliotecas, laboratorios de ciencias y salas de cómputo, y la conexión a electricidad, telefonía, agua potable, desagüe y baños suficientes, son los indicadores que más influyen en el rendimiento. Finalmente, los autores recomiendan que la región debe priorizar la inversión en este tipo de infraestructura, teniendo en cuenta las diferencias observadas entre las escuelas del ámbito rural y urbano, escuelas públicas y privadas y escuelas con estudiantes escasos recursos.

En Duarte et al. (2017) se propuso analizar la infraestructura escolar en América Latina y el Caribe con base en los criterios de suficiencia, equidad y efectividad. El criterio de suficiencia que utilizan los autores se basó en:

- Alumnos que asisten a una escuela con agua y saneamiento suficiente. Escuela con agua potable, alcantarillado, baños en buen estado y recolección de basura.

- Escuela con al menos luz y teléfono.

- Escuela con al menos biblioteca.

- Escuela con al menos dos de los siguientes componentes: oficina del director, oficinas adicionales (de secretaría, de administración, etc.), sala de reuniones para profesores y enfermería.

- Escuela con al menos uno de los siguientes tres componentes: gimnasio, auditorio, campo o cancha deportiva.

- Escuela con todos los componentes en todas las aulas (tiza o marcadores de pizarra; mesa y silla para el profesorado; mesa y silla para cada estudiante).

Con base en lo anterior y la información disponible del TERCE se construyeron seis índices de suficiencia de la infraestructura escolar (se utilizó el método de análisis de componentes principales):

1. Agua y saneamiento: Agua potable; alcantarillado; baños en buen estado; recolección de basura. 
2. Conexión a servicios: Luz eléctrica; teléfono; conexión a internet.

3. Espacios pedagógicos/académicos: Sala de artes y/o música; laboratorio(s) de ciencias; sala de computación; y biblioteca de la escuela.

4. Áreas de oficinas (incluye enfermería): Oficina para el director; oficinas adicionales (de secretaría, de administración, etc.); sala de reuniones para profesores; y enfermería.

5. Espacio de uso múltiple: Gimnasio; auditorio; y campo o cancha deportiva.

6. Equipamiento de aulas: Tiza o marcadores de pizarra; mesa para el y la docente; silla para el y la docente; mesa para cada estudiante; y silla para cada estudiante.

En términos generales, los resultados mostraron que solo la cuarta parte del alumnado evaluado asiste a escuelas que cumplen con todos los índices de suficiencia requeridos. Por ejemplo, en Chile, el 91\% del estudiantado de tercer grado asiste a escuelas que cuentan con cinco o seis índices de suficiencia en infraestructura escolar. En Brasil, Costa Rica, Colombia y Uruguay las cifras son mayor al 50\%. Mientras que en Centroamérica, excepto Costa Rica, los porcentajes de estudiantes que asisten a escuelas con muy pocos índices son muy altos.

Por el lado de la equidad, el criterio de los autores se basa en que no solo es importante la suficiencia de la infraestructura sino que exista igualdad de acceso a todo el estudiantado sin importar aspectos socioeconómicos, ubicación geográfica o pertenencia a grupos étnicos. Los resultados indicaron que cada estudiante que asiste a escuelas en zonas urbanas está en mejores condiciones que aquellos que asisten en zonas rurales; sin embargo, aún en la zona urbana se observan diferencias entre las escuelas públicas y privadas. Un caso especial es el de Chile y Uruguay que presentan menos diferencias en general. Sin embargo, países de Centroamérica como Guatemala, Honduras y Panamá presentan brechas más amplias. Colombia, México y Perú también tienen brechas por nivel socioeconómico y por ubicación geográfica.

Finalmente, respecto a la efectividad, el objetivo fue encontrar la asociación estadística entre la situación de la infraestructura escolar y su rendimiento. Los índices sirvieron de variables explicativas, y así como en Duarte et al. (2011) se utilizó el método de regresión multinivel. Los resultados indicaron que los espacios pedagógicos/académicos y la conexión a servicios de electricidad, teléfono e internet fueron las variables que más afectaron el rendimiento escolar.

En el caso específico de Perú, existen otros estudios como los de Schady y Paxson (1999), Paxson y Schady (2002), Beltrán y Seinfeld (2011), Beltrán y Seinfeld (2013) y Campana, Velasco, Aguirre, y Guerrero (2014) los cuales encontraron resultados similares, en donde se concluye que poner atención en la infraestructura de la escuela tiene efectos positivos sobre el rendimiento escolar.

\section{Regiones naturales, pobreza y educación básica en el Perú}

El país cuenta con 24 departamentos y una provincia constitucional, el Callao (ver Figura 1). Estos departamentos se encuentran en tres regiones naturales: costa, sierra y/o selva. En la Tabla 1, con base en el Instituto Nacional de Estadística e Informática - INEI (2009), se muestra la clasificación de los departamentos por región natural a la que pertenecen, siendo que hay departamentos que se sitúan en más de una región (ver Figura 2). 


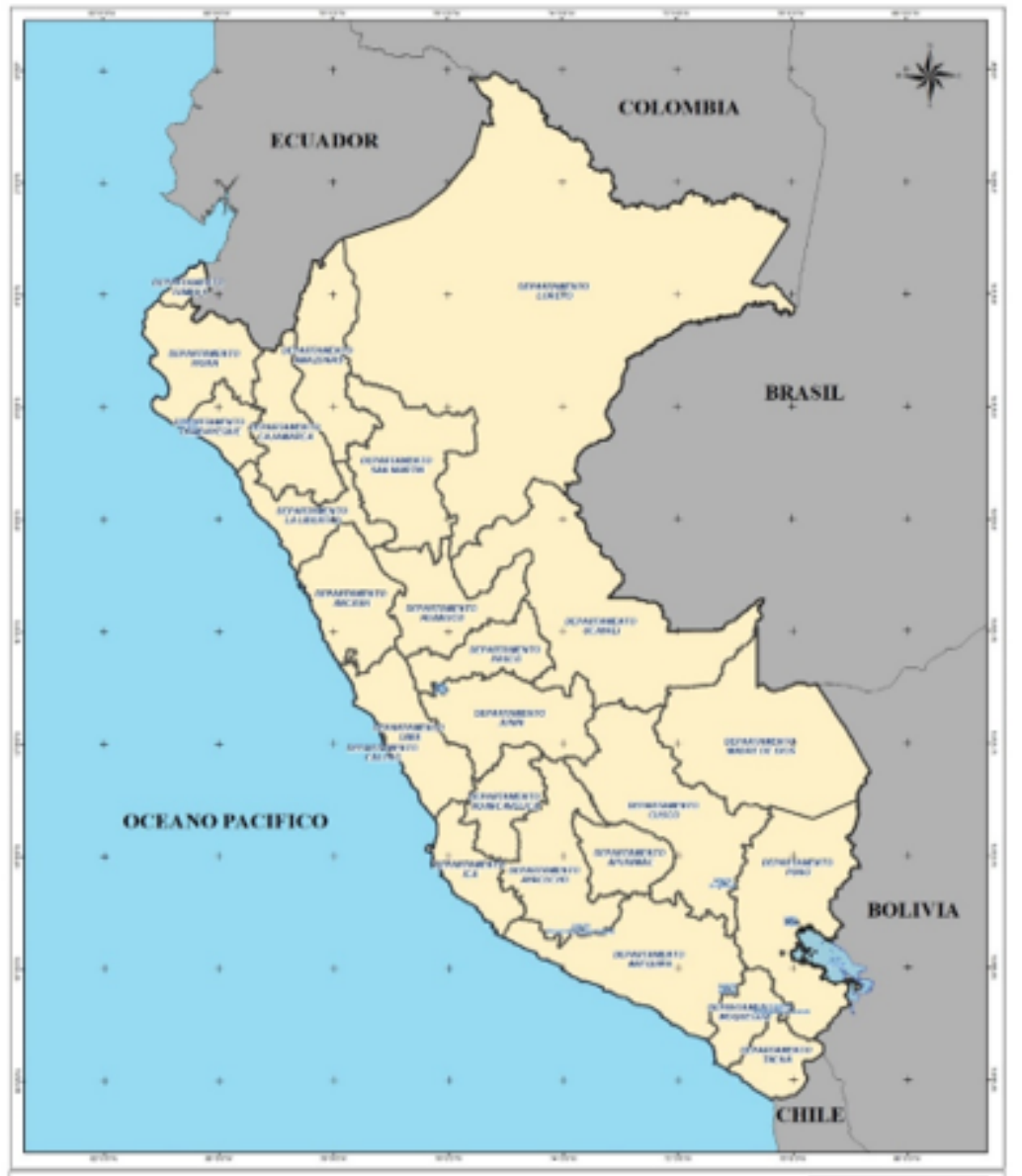

FIGURA 1

Mapa del Perú. División por departamentos y provincia constitucional. Fuente: IDEP (2020).

TABLA 1

Mapa del Perú. División por departamentos y provincia constitucional.

\begin{tabular}{l|l}
\hline Departamento & Región Natural \\
\hline Callao, Ica, La Libertad, Lambayeque, Lima & \\
Metropolitana, Lima Provincias, Moquegua, Piura, & Costa \\
Tacna, Tumbes & \\
\hline Ancash, Apurímac, Arequipa, Ayacucho, & \\
Cajamarca, Huancavelica, Huánuco, Cusco, Junín, & Sierra \\
Pasco, Puno & \\
\hline Amazonas, Loreto, Ucayali, San Martín, Madre de & Selva \\
Dios &
\end{tabular}

Fuente: Elaboración propia con base en INEI (2009)

Nota: La información de región natural se hizo conforme la clasificación general del INEI, siendo que hay departamentos que comparten diferentes regiones. 


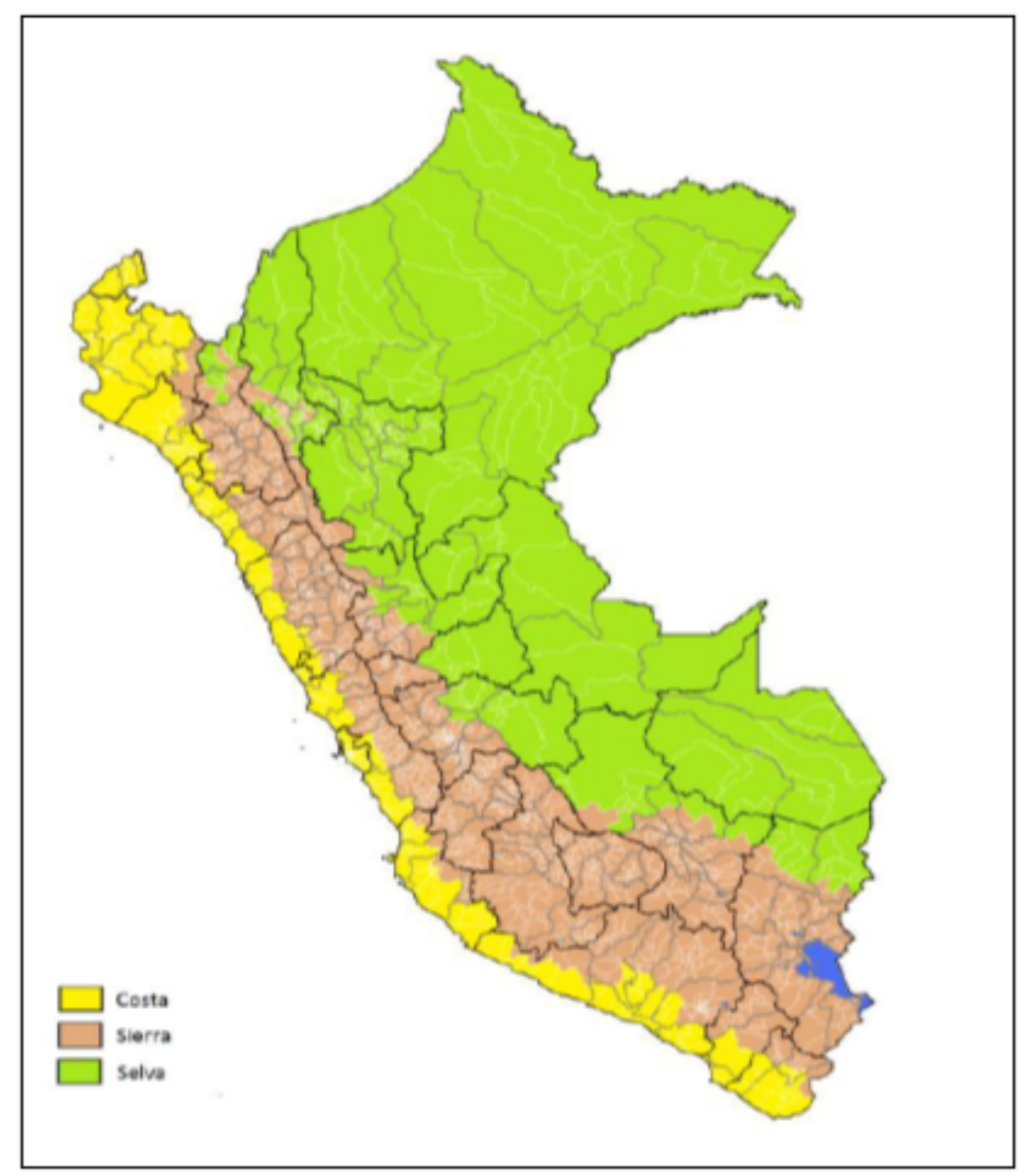

FIGURA 2

Mapa del Perú. Regiones naturales Fuente: BID (2015).

Para el año 2017 la población que se distribuye a lo largo de estos departamentos es mayormente urbana (79,3\%), siendo la región natural costa aquella que más población concentra $(58 \%)$, disminuyendo en el tiempo aquella población que se encuentra en la sierra $(28,1 \%)$ y selva $(13,9 \%)$. La provincia constitucional del Callao tiene el 100\% de población urbana, siguiéndole Lima Metropolitana con 99,9\%, mientras que al extremo se tiene al departamento de Huancavelica con el 69,5\% de población rural, siguiéndole Cajamarca con 64,6\% (INEI, 2018a).

Por otro lado, a pesar de que en cada espacio del territorio peruano se desarrollan distintas actividades económicas que propician el crecimiento y desarrollo del país, persiste el centralismo económico en la capital: Lima (Remy, 2015). Esta situación se refleja en los niveles de pobreza, existiendo diferencias entre departamentos, regiones naturales y dominios geográficos. Según el INEI (2018b) la estimación de la pobreza monetaria para el 2017 en la región Sierra fue del 31,6\%, siguiéndole la Selva con 28,6\% y por último la Costa con $14,4 \%$. Al interior de cada región natural también se pueden observar diferencias; por ejemplo, la sierra rural alcanzó una tasa del 48,7\%, mientras que la zona urbana obtuvo una tasa parecida a la costa urbana $(16,3 \%)$. En conjunto, Lima Metropolitana (donde se encuentra la capital) y el Callao obtuvieron una tasa de 13,3\%, siendo la más baja (ver Figura 3). A nivel departamental, Cajamarca ubicado en la Sierra se encontró en el grupo de la tasa más alta, mientras que Ica en la Costa y Madre de Dios en la Selva en el grupo de la tasa más baja (ver Tabla 2). 


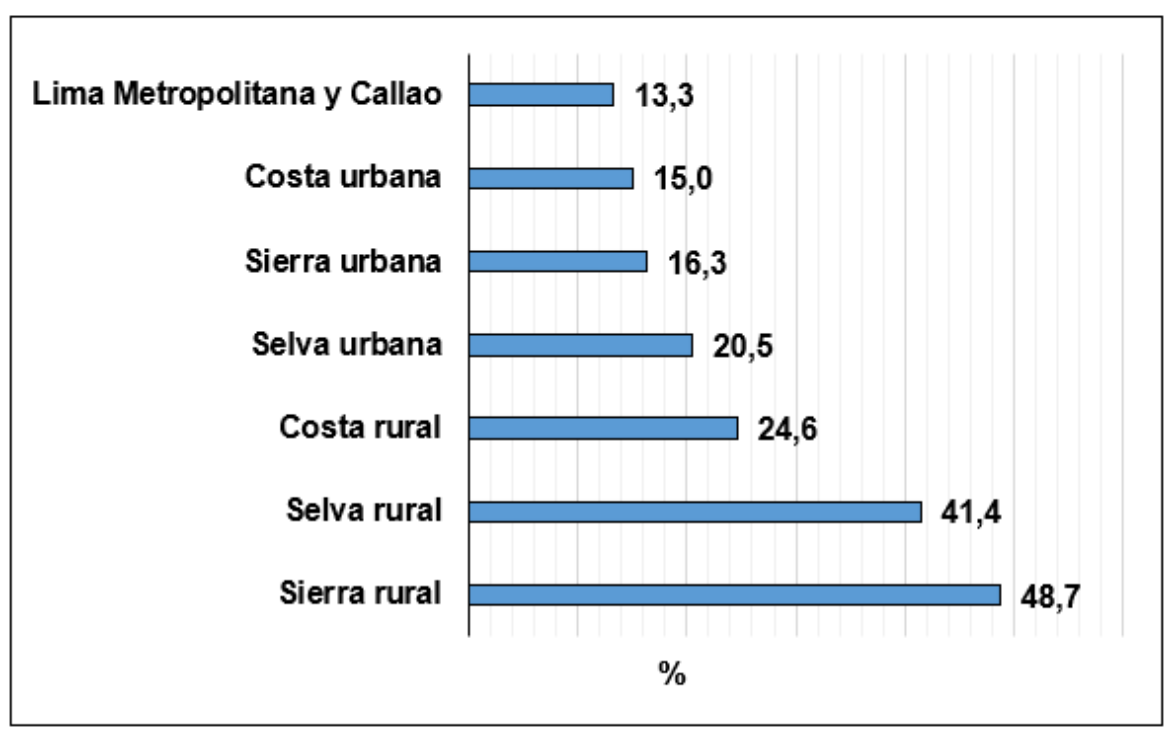

FIGURA 3

Perú: Incidencia de la pobreza monetaria total, según dominios geográficos (2017). Fuente: INEI (2018b)

TABLA 2

Perú: Grupos de departamentos con niveles de pobreza monetaria total, semejantes estadísticamente (2017).

\begin{tabular}{l|l}
\hline Departamentos & Tasa de pobreza \\
\hline Cajamarca & $43,1 \%$ y $52,0 \%$ \\
\hline $\begin{array}{l}\text { Amazonas, Apurimac, Ayacucho, } \\
\text { Huancavelica, Huánuco, Loreto, Pasco, } \\
\text { Puno }\end{array}$ & $33,3 \%$ y $36,8 \%$ \\
\hline $\begin{array}{l}\text { Ancash, Cusco, Junín, La Libertad, Piura } \\
\text { y San Martín }\end{array}$ & $23,0 \%$ y $26,2 \%$ \\
$\begin{array}{l}\text { Arequipa, Lambayeque, Moquegua, } \\
\text { Provincia Constitucional del Callao, } \\
\begin{array}{l}\text { Provincia de Lima, Región Lima, Tacna, } \\
\text { Tumbes y Ucayali }\end{array}\end{array}$ & $12,1 \%$ y $14,6 \%$ \\
\hline \begin{tabular}{l} 
Ica y Madre de Dios \\
\hline
\end{tabular} & $2,1 \%$ y $4,8 \%$ \\
\hline
\end{tabular}

Fuente: INEI (2018b)

Por el lado de los resultados educativos, estos también muestran diferencias por departamentos y regiones. Por ejemplo, según el INEI (2018a), la población censada de 15 y más años sin nivel educativo fue de más del 10\% en los departamentos de la Sierra como Huancavelica, Apurímac, Cajamarca, Ayacucho y Huánuco; mientras que en la Costa, los departamentos Ica, Lima provincias y la provincia constitucional del Callao el porcentaje resultó menor al 2\%. Asimismo, la tasa de población analfabeta en la población de 15 y más años se redujo en la última década, pero aún persisten diferencias entre departamentos. Por ejemplo en la Sierra, los departamentos de Huancavelica (17,7\%), Apurímac (16,8\%), Cajamarca (14,8\%), Ayacucho (13,4\%), Huánuco (12,7\%), Cusco (11,0\%), Puno (10,5\%), Ancash (10,4\%) y Amazonas (10,5\%) quienes el 10\%; mientras que en la Costa, Callao y Lima Metropolitana obtuvieron una tasa menor al 2\%. Asimismo, los resultados de las evaluaciones del Censo Escolar (MINEDU, 2019c) indican que el rendimiento en las áreas de Lectura y Matemática (niños y niñas de segundo grado de primaria) ha mejorado; sin embargo, existe una desigualdad entre departamentos. Por ejemplo, en el caso del rendimiento escolar satisfactorio en Lectura 
para el año 2015, Tacna ocupa el primer lugar con 78,1\% de alumnos, siguiéndole Moquegua con 73,9\%; mientras que Loreto solo alcanzó el 18,1\%. Lo similar en Matemáticas, Tacna alcanzó el 53,5\%, mientras que Loreto solo el 5,8\%. Por último, cabe mencionar que estos resultados son producto del sistema peruano de educación básica, cuyo carácter es obligatorio según el marco legal vigente de la Ley $\mathrm{N}^{\circ} 28044$ (Congreso de la República del Perú, 2003).

En lo que respecta a la infraestructura escolar del país, el MINEDU (2019a) indica que a lo largo del territorio nacional existen 106886 instituciones educativas y 70277 locales escolares (más del 50\% son públicos) que brindan educación básica regular - EBR ${ }^{[3]}$ (Inicial, Primaria y Secundaria) a un total de 7821 050 alumnos. El local escolar se concibe como la edificación o edificaciones construidas sobre un espacio público o privado donde funcionan una o más instituciones educativas, mientras que el aula es el espacio físico donde se desarrolla el proceso de enseñanza y aprendizaje formal (INEI, 2013). La mayoría de los locales escolares se encuentran en el departamento de Lima (15\%), Cajamarca (9,7\%) y Puno (6,1\%); mientras que el menor número se encuentra en Madre de Dios (0,5\%), Tumbes (0,6\%), Moquegua (0,6\%) y Tacna (0,8\%).

La infraestructura escolar es una preocupación preponderante de los hacedores de la política educativa del país, lo que ha conllevado a efectuar diferentes esfuerzos en la última década (Banco Mundial, 2016):

- Primer Censo Nacional de Infraestructura Educativa (2014).

- Implementación del Plan Lima para atender locales escolares con infraestructura en riesgo.

- Implementación del Plan Selva para mejorar la infraestructura educativa en la Amazonía.

- Implementación del aplicativo Wasichay como sistema para declarar y supervisar el gasto en mantenimiento.

- Se impulsaron mecanismos de obras públicas involucrando la participación tanto pública como privada.

Asimismo, se desarrolló el Plan Nacional de Infraestructura Educativa (PNIE) al 2025, el cual tiene por objetivo "mejorar la condición, capacidad, gestión y sostenibilidad de la infraestructura educativa pública para avanzar hacia una educación de calidad para todos” (Banco Mundial, 2017, p.1). El Plan contempla cuatro objetivos específicos:

1. Asegurar condiciones básicas de seguridad y funcionalidad en la infraestructura educativa existente.

2. Ampliar la capacidad de la infraestructura educativa para atender de manera óptima la demanda aún no cubierta.

3. Fortalecer la gestión de la infraestructura educativa en todos sus niveles.

4. Garantizar la sostenibilidad de la infraestructura educativa.

\section{Metodología}

La investigación tiene un alcance descriptivo cuantitativo y utiliza datos transversales y longitudinales. La unidad de análisis corresponde a las características de la infraestructura escolar de las escuelas en los departamentos del Perú, cuya información proviene de la base de datos Estadísticas de Calidad Educativa ESCALE del (MINEDU, 2019b). Con el objetivo de resumir dichas características se construyó un índice sintético de suficiencia de infraestructura escolar ${ }^{[4]}$ denominado Índice de Infraestructura Escolar- IE. Para ello se aplicó un Análisis de Componentes Principales (ACP) el cual es un "método geométrico de carácter descriptivo [cuyo] objetivo es descubrir la estructura subyacente en un conjunto de n individuos estudiados bajo una serie de p variables cuantitativas" (Lévy y Varela, 2003, p. 329). El IE se construyó para los años 2005 y 2017 con el propósito de comparar tendencias. 
El criterio de suficiencia, que se basó en el planteamiento de Duarte et al. (2017) y Beltrán y Seinfeld (2013) ${ }^{[5]}$, incluyó cuatro indicadores:

1. Escuelas públicas con todas sus aulas en buen estado (ABE).

2. Escuelas públicas con suficientes pizarras (SP).

3. Escuelas públicas con suficientes carpetas (SC).

4. Escuelas públicas con los tres servicios básicos (TSB).

La descripción de cada uno se muestra en la Tabla 3.

TABLA 3

Indicadores de Suficiencia de la Infraestructura Escolar en Educación Básica

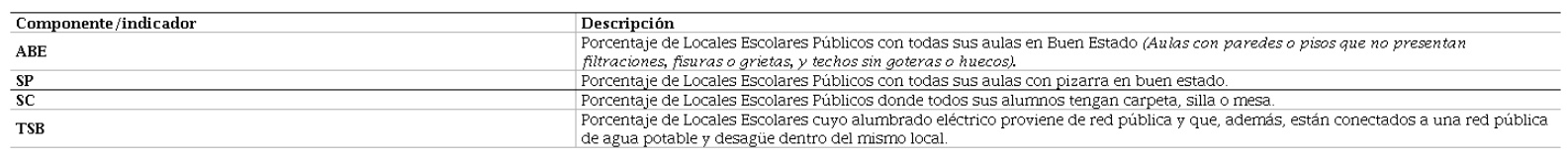

Fuente: Elaboración propia con base en MINEDU (2019b)

La representación matemática del ACP indica que las puntuaciones factoriales correspondientes a cada observación tienen una estructura de la forma siguiente (Lévy y Varela, 2003):

$$
\begin{gathered}
c_{h i}=c_{h 1} x_{1 i}+c_{h 2} x_{2 i}+c_{h 3} x_{3 i}+\cdots_{. .}+c_{h p} x_{p i} \\
h=1,2, \ldots p \\
i=1,2, \ldots, n
\end{gathered}
$$

Es decir, hay una componente por cada variable que conforma el índice, por lo que para el IEC se obtuvieron cuatro componentes con n igual a 26 (23 departamentos, una provincia constitucional y el departamento de Lima se dividió en Lima Metropolitana y Provincias) y se eligió la que mayor varianza explica (componente 1). La estructura del IEC resultó la siguiente:

$$
\begin{gathered}
c_{h i}=c_{h 1} x_{1 i}+c_{h 2} x_{2 i}+c_{h 3} x_{3 i}+c_{h 4} x_{4 i} \\
h=1,2, \ldots \\
i=1,2, \ldots, 26
\end{gathered}
$$

Los resultados del análisis permitió jerarquizar a los departamentos conforme el valor del IEC. A través de cuantiles se pudo obtener rangos de suficiencia: Alto, Medio, Bajo y Muy Bajo.

\section{Resultados}

Los primeros resultados mostraron que a nivel nacional los componentes de suficiencia Escuelas públicas con todas sus aulas en buen estado - ABE y Escuelas públicas con los tres servicios básicos - TSB son los que más problemas han tenido entre los años 2005 y 2017, pues no alcanzan ni el 50\%; por otro lado, el componente Escuelas públicas con suficientes carpetas - SC es el que mejor se ha comportado, alcanzando un porcentaje de más del 70\%, siguiéndole Escuelas públicas con suficientes pizarras - SP (ver Figura 4). 


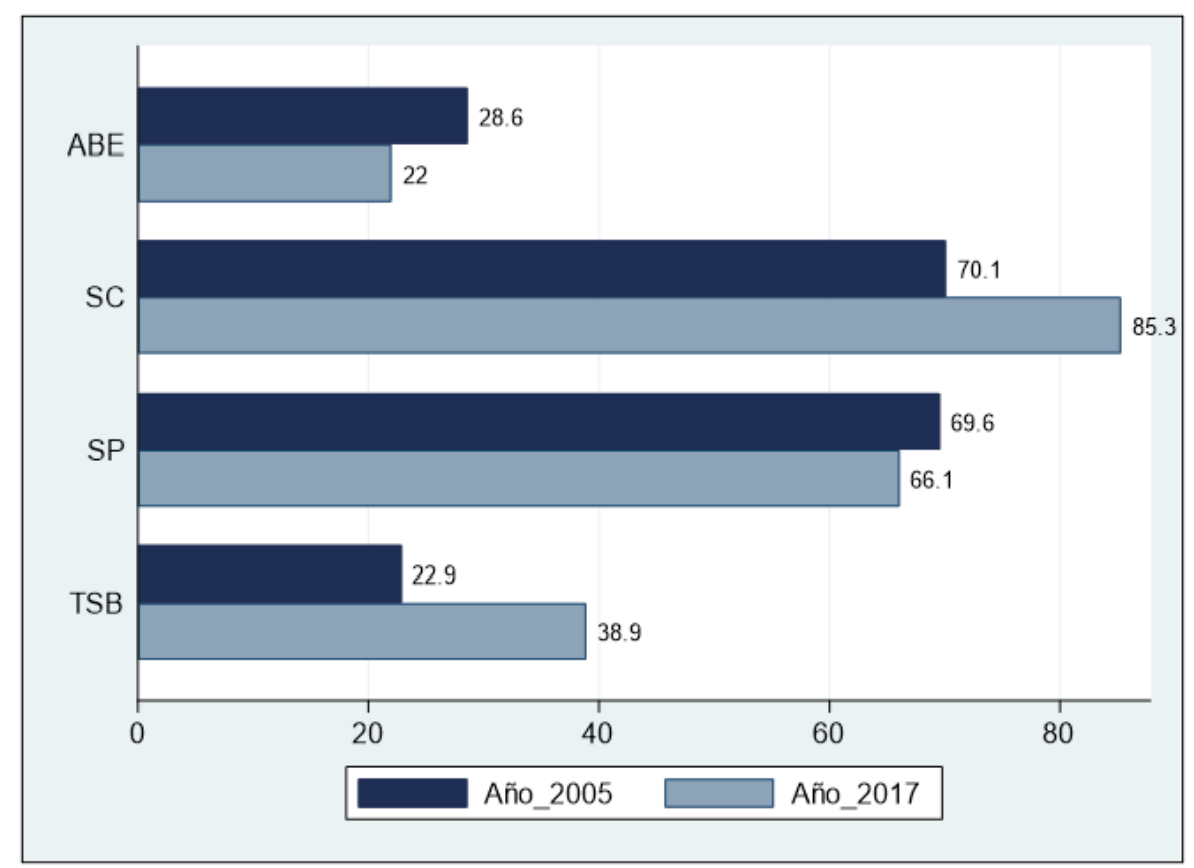

FIGURA 4

Perú: Porcentaje de escuelas con infraestructura escolar suficiente (2005 y 2017) Fuente: Elaboración propia con base en MINEDU (2019b)

A nivel departamental, el componente que obtuvo una mayor desigualdad fue Escuelas públicas con los tres servicios básicos - TSB, pues la desviación estándar estimada resultó por encima de los otros componentes de suficiencia, con un cambio prácticamente no significativo entre los años de análisis (ver Figura 5).

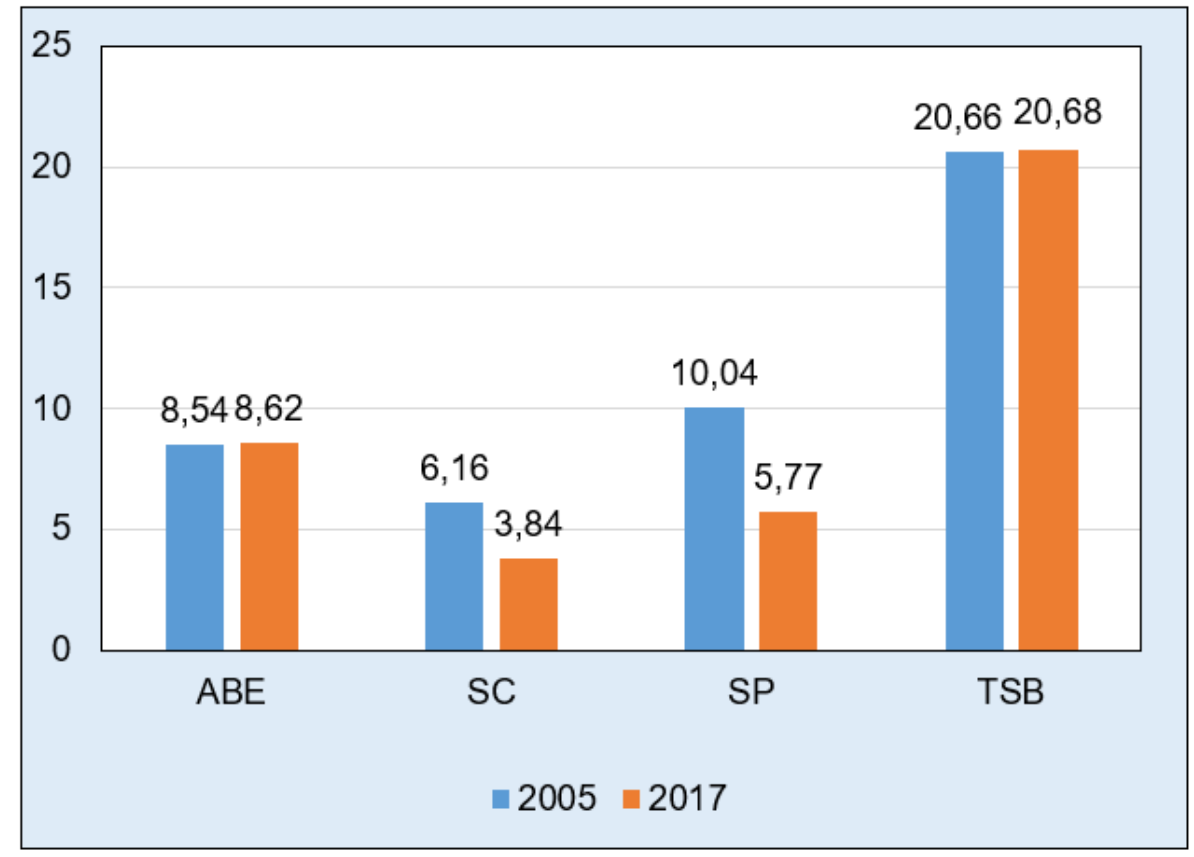

FIGURA 5

Desviación estándar de los componentes de suficiencia de la infraestructura escolar (2005 y 2017). Fuente: Elaboración propia con base en MINEDU (2019b) 
En la Figura 6 se puede observar que ningún departamento obtuvo valores sobresalientes sobre el total de los componentes. Lo que se observó es cada uno de ellos destacó en ciertas características. Por ejemplo, Tacna destacó por obtener el mejor valor en ABE (50.1\%), en SP (81.4\%) y en SC (93.6\%); mientras que Lima Metropolitana, Callao, Ica, Tumbes y Moquegua destacaron por tener los mejores valores en TSB (por encima del 50\%); por el contrario, Loreto y Ucayali no alcanzan ni el 20\% en dicha característica. Apurímac obtuvo los valores más bajos en $\mathrm{ABE}$ (10.7\%), siguiéndole Puno (12.4\%).

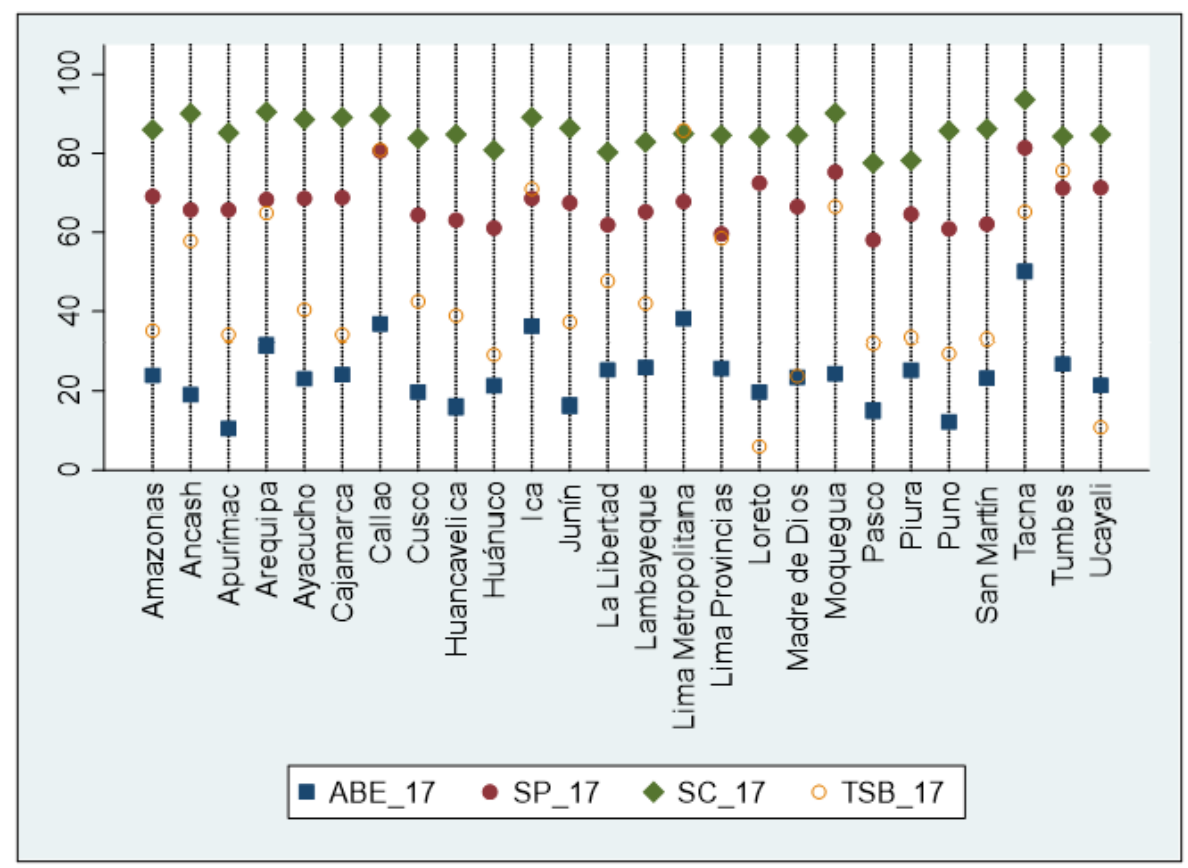

FIGURA 6

Porcentaje de escuelas con infraestructura escolar suficiente por departamentos (2017).

Fuente: Elaboración propia con base en MINEDU (2019b)

El resultado del IE para los años 2005 y 2017 se muestran en las Figuras 7 y 8 respectivamente ${ }^{[6]}$. En el año 2005, el departamento de Ica (Costa) obtuvo el mayor valor del IE, mientras que Ayacucho (Sierra) el menor. Para el año 2017, el IE más alto le corresponde al departamento de Tacna (Costa); mientras que Pasco (Sierra) obtuvo el menor. Una clasificación más específica se muestra en las Tablas 4 y 5 en donde se ha ordenado los departamentos por rangos de IE obtenido. 


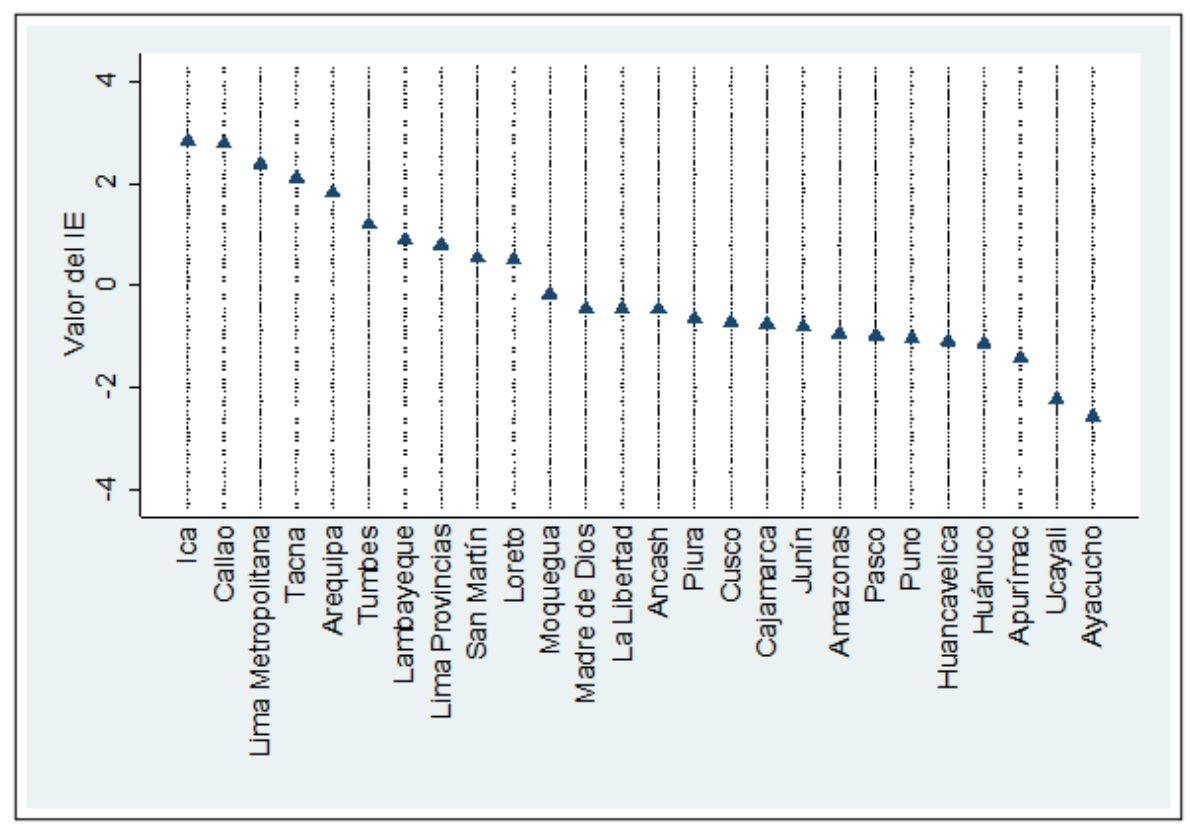

FIGURA 7

Jerarquización del IE por departamentos (año 2005)

Fuente: Elaboración propia con base en MINEDU (2019b)

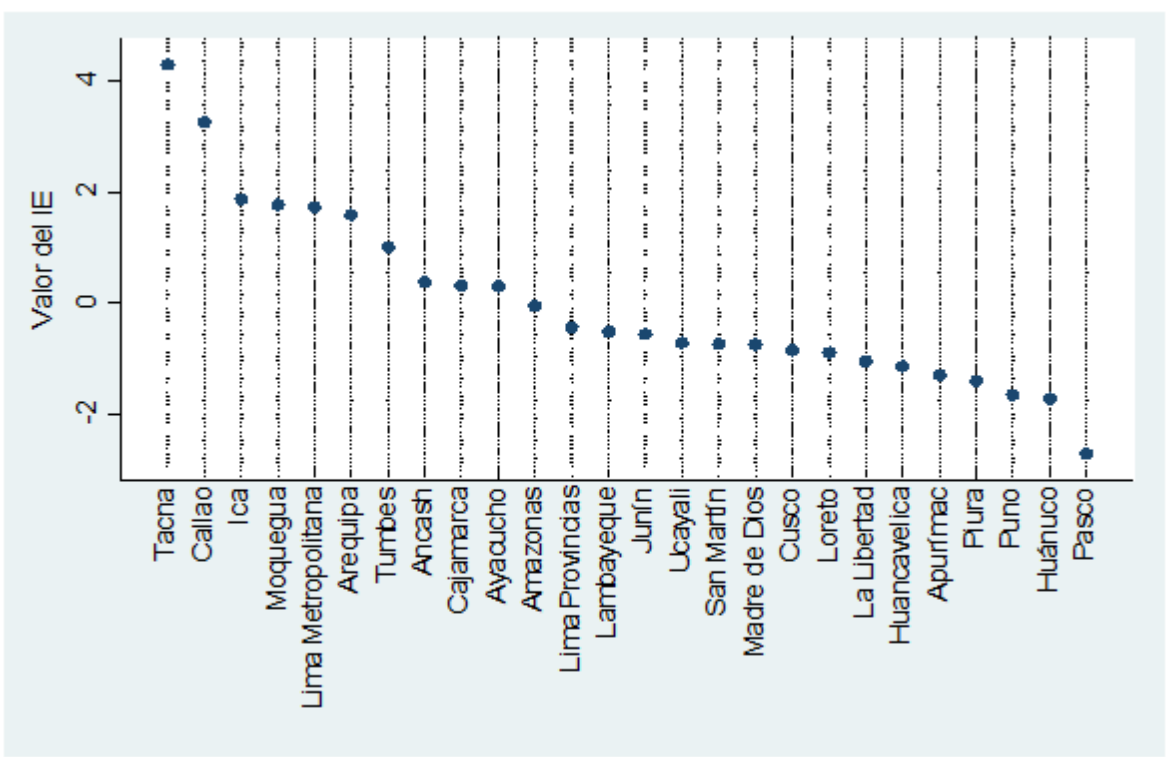

FIGURA 8

Jerarquización del IE por departamentos (año 2017)

Fuente: Elaboración propia con base en MINEDU (2019b)

En el año 2005 Ica y Ayacucho obtuvieron un índice Alto y Muy Bajo respectivamente, mostrando el contraste entre la región Costa y Sierra. Callao, Lima Metropolitana, Tacna, Arequipa y Tumbes obtuvieron un índice Alto; mientras que Lambayeque, Lima provincias, San Martín, Loreto, Moquegua, Madre de Dios y La Libertad obtuvieron un valor Medio. El índice Bajo lo obtuvieron Ancash, Piura, Cusco, Cajamarca, Junín y Amazonas; y en la categoría Muy Bajo se ubicaron Pasco, Puno, Huancavelica, Huánuco, Apurímac y Ucayali (ver Tabla 4). 
Tabla 4

\begin{tabular}{|c|c|c|}
\hline Rango & Departamento & IE \\
\hline \multirow{6}{*}{ Alto } & Ica & 2.84 \\
\hline & Callao & 2.77 \\
\hline & Lima Metropolitana & 2.38 \\
\hline & Tacna & 2.11 \\
\hline & Arequipa & 1.81 \\
\hline & Tumbes & 1.19 \\
\hline \multirow{7}{*}{ Medio } & Lambayeque & 0.9 \\
\hline & Lima Provincias & 0.8 \\
\hline & San Martín & 0.55 \\
\hline & Loreto & 0.51 \\
\hline & Moquegua & -0.17 \\
\hline & La Libertad & -0.46 \\
\hline & Madre de Dios & -0.46 \\
\hline \multirow{6}{*}{ Bajo } & Ancash & -0.47 \\
\hline & Piura & -0.64 \\
\hline & Cusco & -0.72 \\
\hline & Cajamarca & -0.76 \\
\hline & Junín & -0.81 \\
\hline & Amazonas & -0.94 \\
\hline \multirow{7}{*}{ Muy Bajo } & Pasco & -0.97 \\
\hline & Puno & -1.03 \\
\hline & Huancavelica & -1.09 \\
\hline & Huánuco & -1.13 \\
\hline & Apurimac & -1.42 \\
\hline & Ucayali & -2.23 \\
\hline & Ayacucho & -2.56 \\
\hline
\end{tabular}

Para el año 2017 los departamentos de Tacna, Callao, Ica, Lima Metropolitana y Arequipa continuaron con un índice Alto, incorporándose a este rango el departamento de Moquegua. Tumbes aparece ahora dentro de la categoría de departamentos con un valor del índice Medio junto a Ancash, Cajamarca, Ayacucho, Amazonas, Lima provincias y Lambayeque (estos dos últimos conservaron su categoría respecto al año 2005). Los departamentos que obtuvieron un índice Bajo fueron Junín, Ucayali, San Martín, Madre de Dios, Cusco y Loreto; siendo que Junín y Cusco se mantuvieron en dicha categoría. San Martín, Loreto y Madre de Dios pasaron de Medio a Bajo. En el caso del grupo de departamentos con índice Muy Bajo resaltaron los casos de La Libertad y Piura, pues pasaron de un índice Medio y Bajo respectivamente a esta categoría; mientras que Huancavelica, Apurímac, Huánuco, Puno y Pasco se mantuvieron con un índice Muy Bajo (ver Tabla 5). 
TABLA 5

IE por departamentos (2017)

\begin{tabular}{|c|c|c|}
\hline Rango & Departamento & IE \\
\hline \multirow{6}{*}{ Alto } & Tacna & 4.29 \\
\hline & Callao & 3.25 \\
\hline & ICa & 1.87 \\
\hline & Moquegua & 1.77 \\
\hline & Lima Metropolitana & 1.72 \\
\hline & Arequipa & 1.59 \\
\hline \multirow{7}{*}{ Medio } & Tumbes & 1.01 \\
\hline & \begin{tabular}{|l|} 
Ancash \\
\end{tabular} & 0.38 \\
\hline & Cajamarca & 0.31 \\
\hline & Ayacucho & 0.3 \\
\hline & Amazonas & -0.05 \\
\hline & Lima Provincias & -0.44 \\
\hline & Lambayeque & -0.52 \\
\hline \multirow{6}{*}{ Bajo } & Junin & -0.56 \\
\hline & Ucayali & -0.71 \\
\hline & San Martín & -0.73 \\
\hline & Madre de Dios & -0.75 \\
\hline & Cusco & -0.85 \\
\hline & Loreto & -0.9 \\
\hline \multirow{7}{*}{ Muy Bajo } & La Libertad & -1.05 \\
\hline & Huancavelica & -1.14 \\
\hline & Apurimac & -1.29 \\
\hline & Piura & -1.41 \\
\hline & Puno & -1.65 \\
\hline & Huánuco & -1.72 \\
\hline & Pasco & -2.72 \\
\hline
\end{tabular}

Fuente: Elaboración propia con base en MINEDU (2019b)

En las Figuras 9 se puede observar que para el año 2005 el rango Alto tiene un mayor número de departamentos ubicados en la Costa; mientras que los valores Bajo y Muy Bajo agrupan un mayor número en la Sierra. Más de diez años después la tendencia es la misma (ver Figura 10). 


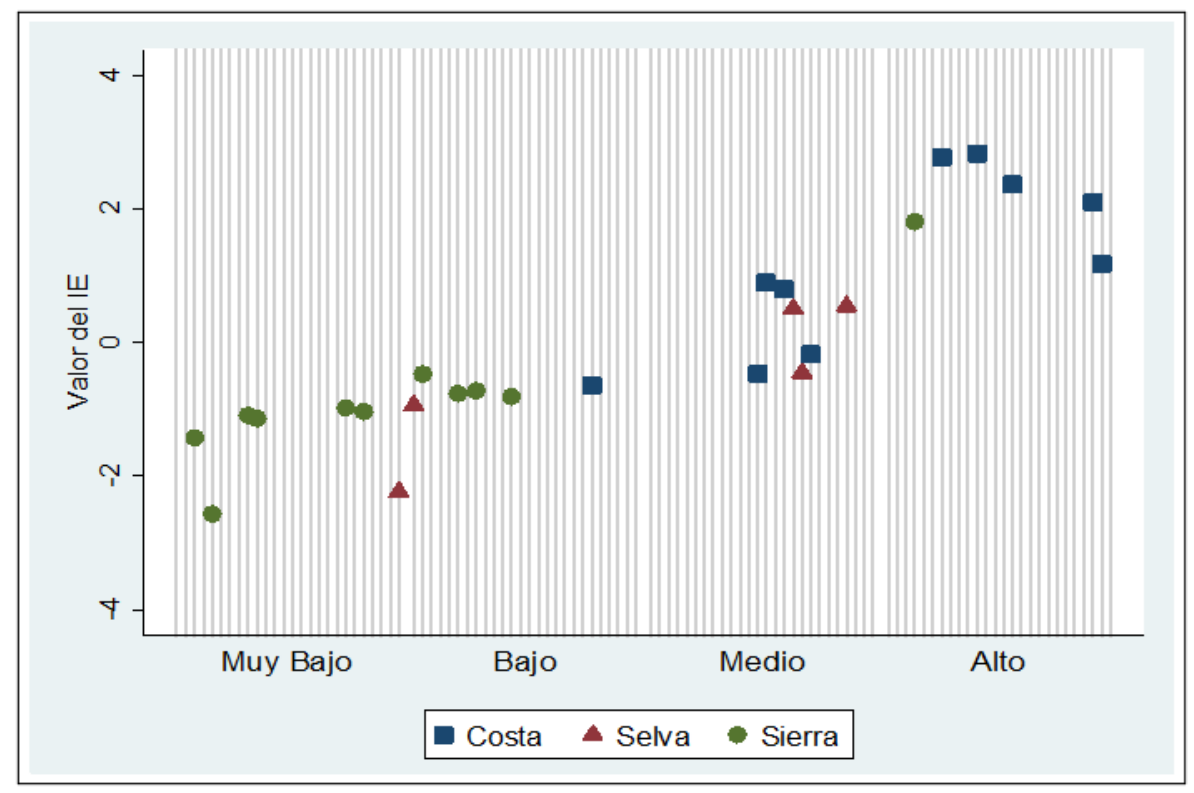

Figura 9

IEC por regiones naturales (año 2005)

Fuente: Elaboración propia con base en MINEDU (2019b)

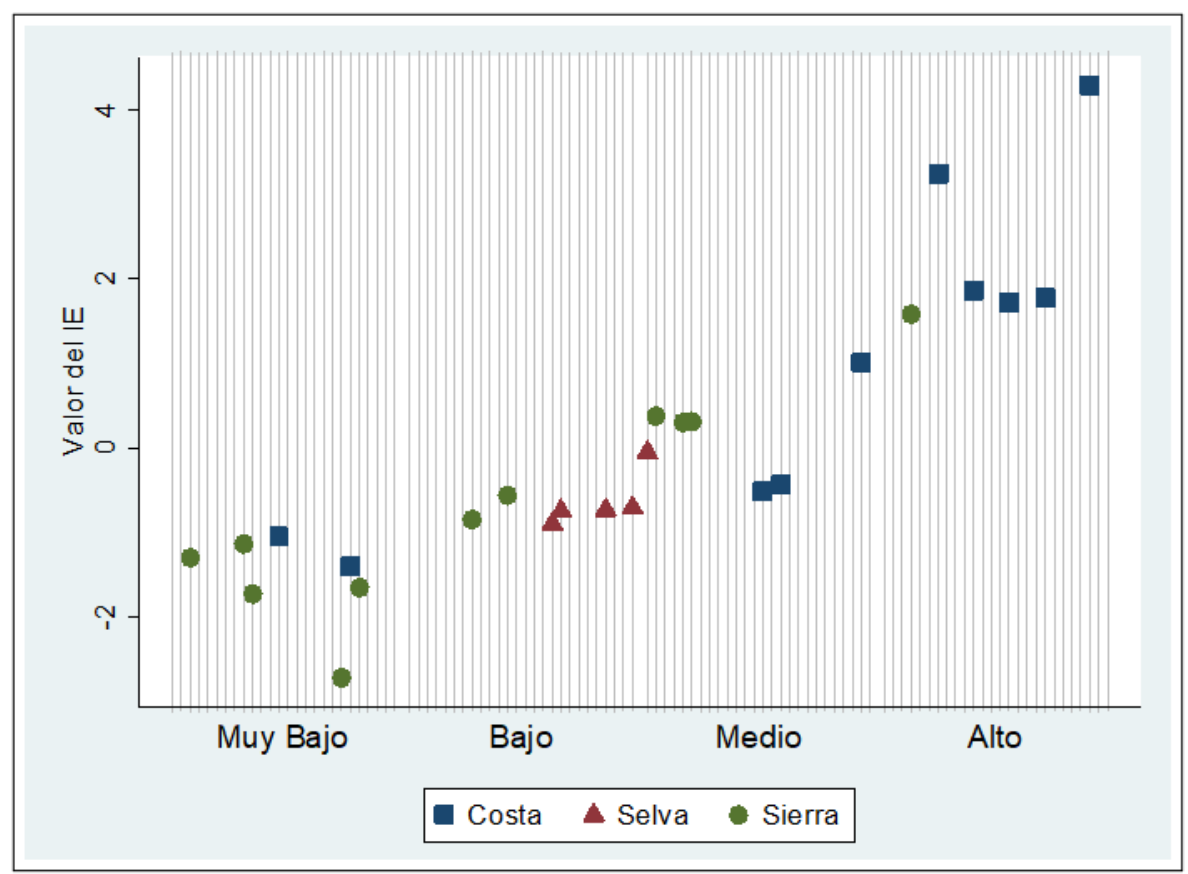

FIGURA 10

IE por regiones naturales (año 2017)

Fuente: Elaboración propia con base en MINEDU (2019b)

En el caso del departamento de Lima, donde se encuentra la capital política y económica del país, sobresale la diferencia que existe entre las zonas de Lima Metropolitana y Lima Provincias, pues en ambos años de análisis esta última obtuvo un IE Medio, mientras que Lima Metropolitana obtuvo un IE Alto.

Finalmente, en las Figuras 11 y 12 se presentan a través de un mapa los resultados del IE por departamentos. Nótese la mayor concentración de IE Alto en la región Costa. 


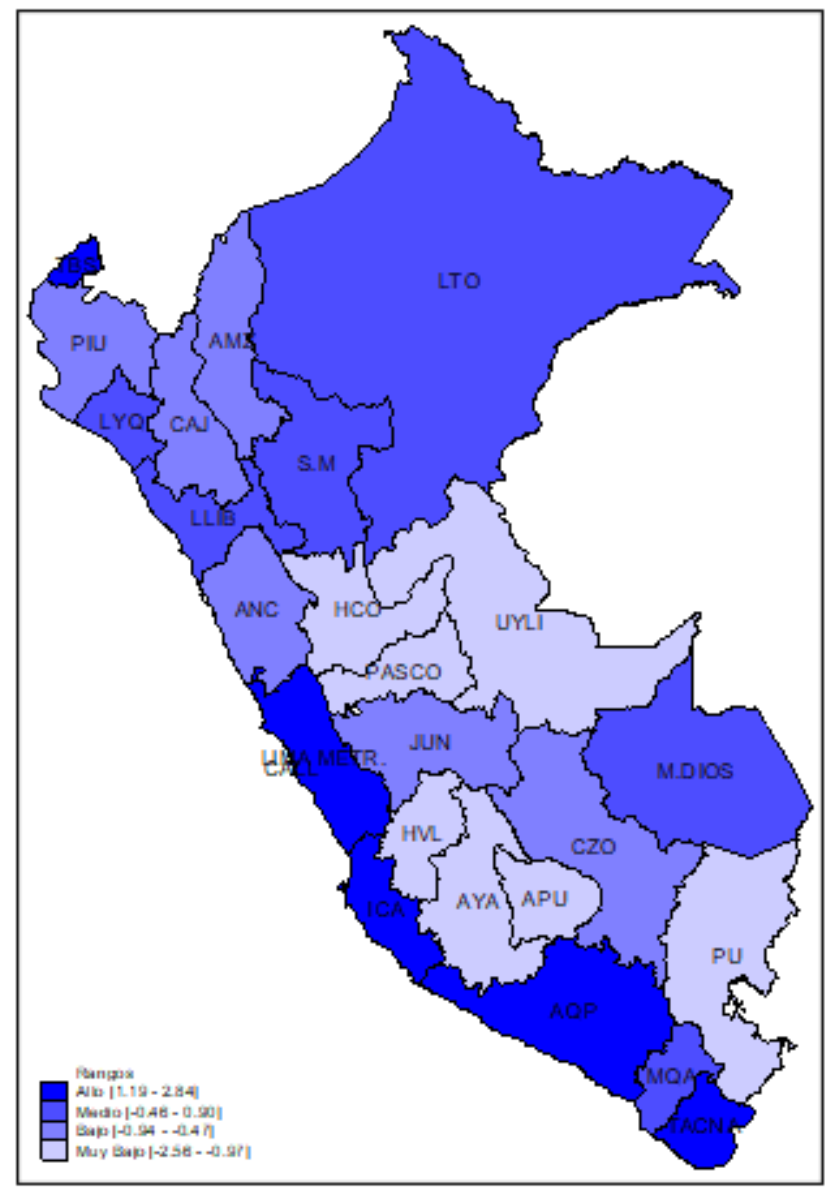

FIGURA 11

IE por rangos y departamentos (año 2005)

Nota: Lima Metropolitana se muestra en todo el departamento de Lima.

Fuente: Elaboración propia con base en MINEDU (2019b) 


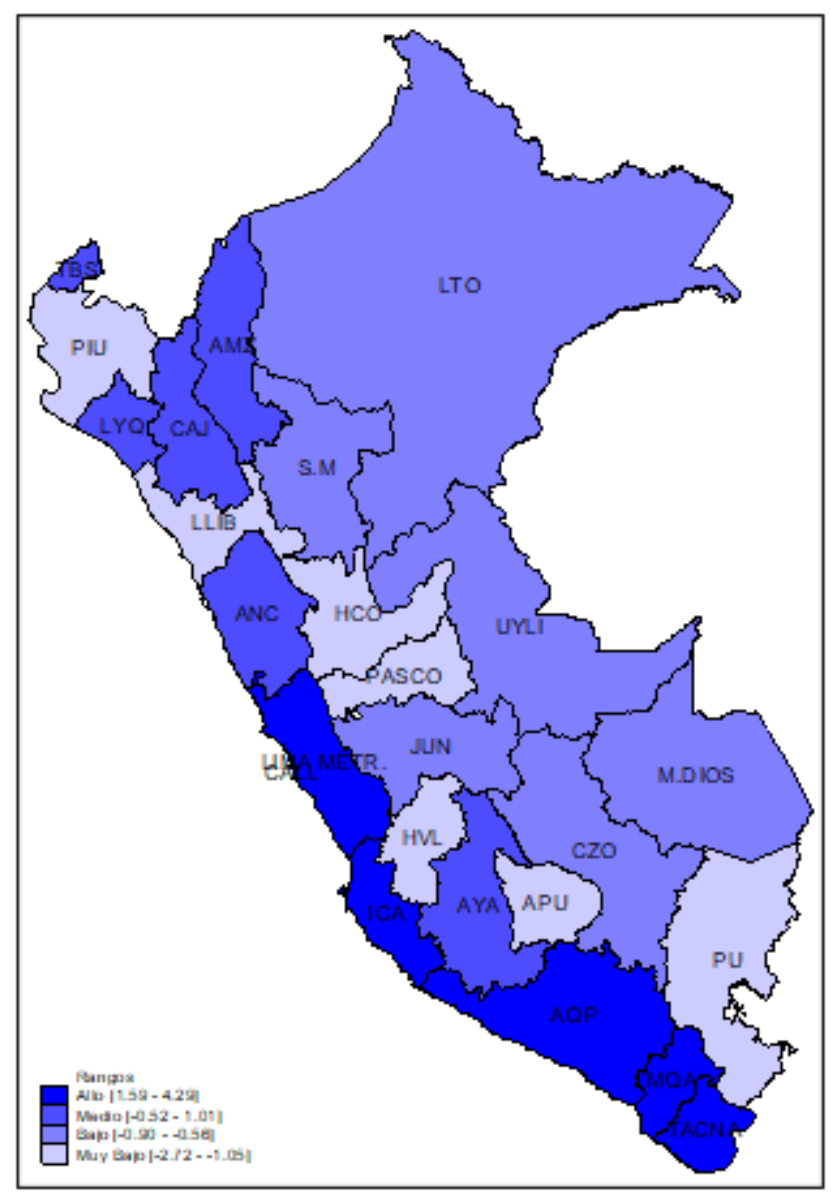

FIGURA 12

IE por rangos y departamentos (año 2017)

Nota: Lima Metropolitana se muestra en todo el departamento de Lima Fuente: Elaboración propia con base en MINEDU (2019b)

\section{CONCLUSIONES Y RECOMENDACIONES}

El objetivo de la investigación fue describir la suficiencia y equidad de la infraestructura escolar en el Perú, para ello se elaboró un índice sintético el cual mostró que una suficiente infraestructura escolar es relativamente mejor en la región natural Costa y peor en la Sierra, tendencia que parece no haber cambiado en más de diez años. Esto conlleva a presenciar un problema de acceso a infraestructura escolar suficiente entre departamentos y regiones naturales. Los resultados comprueban la hipótesis planteada, la cual puede tener diferentes causas que se deberán definir y evaluar en investigaciones futuras.

De forma preliminar, estas causas se pueden atribuir a las condiciones de pobreza que atraviesan los departamentos y regiones, en donde la región Sierra es la más afectada. Sin embargo, también se observó que las diferencias ocurren dentro de los departamentos, tal es el caso de Lima, cuyos valores del índice ubicaron a la zona metropolitana en el rango Alto y a la zona de provincias en el Medio. En lo sucesivo se deberá reflexionar sobre esto.

Por otro lado, los departamentos que destacaron en ambos años de análisis fueron Ica, Tacna, Callao, Lima Metropolitana y Arequipa, pues alcanzaron un valor de IE Alto. Sin embargo, también se observó que la suficiencia de la infraestructura tiene problemas per se, ya que los componentes del índice como aulas en Buen Estado o aulas con Acceso a los Tres Servicios Públicos, presentaron porcentajes en general deficientes (a excepción de Lima Metropolitana). 
Finalmente, a manera de recomendación, por un lado es de conocimiento que el gobierno central está poniendo atención en este asunto; sin embargo, también será importante la participación de los gobiernos regionales y municipales. En este sentido, deben existir condiciones para que los gobiernos locales se involucren en la construcción y mantenimiento de infraestructura escolar, más allá de los aspectos que el marco legal indica, es decir, los gobernadores, alcaldes y también los administradores de las escuelas deben tener clara la importancia de una infraestructura escolar suficiente y equitativa. Se espera que esta investigación forme parte de la evidencia empírica sobre la desigualdad de los insumos educativos en el Perú e incite a otros investigadores a preocuparse por el tema.

\section{REFERENCIAS}

Actis di Pasquale, E. (2015). La elaboración de índices sintéticos de bienestar social. Validación teórica y empírica del método de agregación/ponderación. $12^{\circ}$ Congreso Nacional de Estudios del Trabajo. Buenos Aires, Argentina

Banco Mundial. (2016). Perú: infraestructura educativa al 2025. Perú: Banco Mundial. Recuperado de http://reposi torio.minedu.gob.pe/handle/123456789/5087

Banco Mundial. (2017). Propuesta de Plan Nacional de Infraestructura educativa en Perú al 2025: Metodología de costeo de las intervenciones. Washington D.C.: Banco Internacional de Reconstrucción y Fomento/Banco Mundial.

Barrett, P., Treves, A., Shmis, T., Ambasz, D. y Ustinova, M. (2019). The Impact of School Infrastructure on Learning. A Synthesis of the Evidence. Washington, DC: International Bank for Reconstruction and Development / The World Bank.

Beltrán, A., y Seinfeld, J. (2011). Hacia una educación de calidad: La importancia de los recursos pedagógicos en el rendimiento escolar. Lima, Perú: Universidad del Pacífico - Consorcio de Investigación Económica y Social.

Beltrán, A. y Seinfeld, J. (2013). La trampa educativa en el Perú: Cuando la educación llega a muchos, pero sirve a pocos. Lima, Perú: Universidad del Pacífico.

BID. (2015). Perfil de Riesgo por Inundaciones en Perú. Informe Nacional. División de Medio Ambiente, Desarrollo Rural y Gestión del Riesgo por Desastres.

Cameron, S., Daga, R., y Outhred, R. (2018). Setting out a conceptual framework for measuring equity in learnin.. En UNESCO, Handbook on Measuring Equity in Education (pp. 16-45). Quebec, Canadá: UNESCO Institute for Statistics.

Campana, Y., Velasco, D., Aguirre, J., y Guerrero, E. (2014). Inversión en infraestructura educativa: una aproximación a la medición de sus impactos a partir de la experiencia de los Colegios Emblemáticos. Lima, Perú: CIES.

Carnoy, M. (2006). Economía de la Educación. España: Editorial UOC.

Congreso de la República del Perú. (2003). Ley General de Educación. Ley No 28044. Lima, Perú: Congreso de la República del Perú.

Duarte, J., Gargiulo, C. y Moreno, M. (2011). Infraestructura escolar y aprendizajes en la educación básica latinoamericana: Un análisis a partir del SERCE. Washington D.C.: Banco Interamericano de Desarrollo.

Duarte, J., Jaureguiberry, F. y Racimo, M. (2017). Suficiencia, equidad y efectividad de la infraestructura escolar en América Latina según el TERCE. Santiago: BID - Oficina Regional de Educación para América Latina y el Caribe de la UNESCO.

Formichella, M. M. (2011). Análisis del concepto de equidad educativa a la luz del enfoque de las capacidades de Amartya Sen. Revista Educación, 35(1), 15-34.

Fornari, L. (2012). Diseño de escuelas secundarias en Italia. En Banco Interamericano de Desarrollo (Comp.), Aprendizaje en las escuelas del siglo XXI. Hacia la construcción de escuelas que promueven el aprendizaje, ofrecen seguridad y protegen el medio ambiente. (pp. 63 - 72). Washington D.C.: Banco Interamericano de Desarrollo División de Educación.

Harbinson, R. y Hanushek, E. (1992). Educational Performance of the Poor: Lessons from Rural Northeast Brazil. (3º ed.). Washington D.C.: Word Bank. 
Hille, R. (2012). Diseño de edificios para preescolar y jardín de infantes: Precedentes arquitectónicos. En Banco Interamericano de Desarrollo (Comp.), Aprendizaje en las escuelas del siglo XXI. Hacia la construcción de escuelas que promueven el aprendizaje, ofrecen seguridad y protegen el medio ambiente. (pp. 19-34). Washington D.C.: Banco Interamericano de Desarrollo. División de Educación.

INEI. (2009). Perú: Estimaciones y Proyecciones de Población por Departamento, Sexo y Grupos Quinquenales de Edad, 1995-2025. Perú: El Perú avanza Recuperado de http://proyectos.inei.gob.pe/web/biblioineipub/bancopub/E st/Lib0846/libro.pdf

INEI. (2013). Seguimiento a los factores que Influyen en los logros de aprendizaje. Glosario de Términos. Lima, Perú: Instituto Nacional de Estadística e Informática.

INEI. (2018a). Perú: Perfil sociodemográfico. Informe Nacional. Recuperado de https://bit.ly/3aeNPaN

INEI. (2018b). Evolución de la pobreza monetaria 2007-2017. Perú: Instituto Nacional de Estadística e Informática. Recuperado de https://bit.ly/3epm82p

IDEP. (2020, Marzo 17). Mapa Departamental del Perú. Recuperado de https://www.idep.gob.pe/geovisor/VisorD eMapas/

Jain, C., y Prasad, N. (2018). Quality of Secondary Education in India. Concepts, Indicators, and Measurement. Singapore: Springer Nature Singapore Pte Ltd.

Lévy J. y Varela, J. (2003). Análisis Multivariable para las Ciencias Sociales. Madrid: Pearson Educación.

MINEDU. (2019a). Estadistica de la Calidad Educativa. Recuperado de http://escale.minedu.gob.pe/magnitudes

MINEDU. (2019b). Estadistica de la Calidad Educativa. Recuperado de http://escale.minedu.gob.pe/ueetendencias

MINEDU. (2019c).SICRECE. Recuperado de https://sistemas15.minedu.gob.pe:8888/evaluacion_censal_publico

Mondéjar, J.y Vargas, M. (2008). Indicadores sintéticos: una revisión de los métodos de agregación. Economía, Sociedad $y$ Territorio, 8(27), 656-585.

O’Donnell, S. (2012). El diseño de las escuelas primarias. En Banco Interamericano de Desarrollo (Comp.), Aprendizaje en las escuelas del siglo XXI. Hacia la construcción de escuelas que promueven el aprendizaje, ofrecen seguridad y protegen el medio ambiente. (pp. 35-48). Washington D.C.: Banco Interamericano de Desarrollo División de Educación.

Paxson, C. y Schady, N. (2002). The Allocation and Impact of Social Funds: Spending on School Infrastructure in Peru. The World Bank Economic Review, (16-2), 297-319.

Quesada, M. (2019). Condiciones de la infraestructura educativa en la región pacífico central: los espacios escolares que promueven el aprendizaje en las aulas. Revista Educación, 43(1), 293-311. Recuperado de https://www.red alyc.org/articulo.oa?id=440/44057415023

Remy, M. I. (2015). Desigualdad territorial en el Perú. Reflexiones preliminares. Lima, Perú: Instituto de Estudios Peruanos.

Schady, N. y Paxson, C. (1999). Do School Facilities Matter? The Case of the Peruvian Social Fund (FONCODES). Washington D.C.: World Bank.

UNESCO. (2014). Comparación de resultados del Segundo y Tercer Estudio comparativo y explicativo: SERCE y TERCE, 2006-2013. Recuperado de https://unesdoc.unesco.org/ark:/48223/pf0000244239

Vences, J. (2014). Construcción de un índice compuesto y aproximación para medir los cambios en el tiempo. Realidad, datos y espacio, 5(2), 104-115.

\section{Notas}

[1] El autor quiere agradecer a los revisores pares por sus observaciones pertinentes y de manera especial al Dr. Ignacio Llamas por la idea inicial, a la Dra. Hortensia Moreno por la ayuda metodológica y a la Mtra. Ana González por la corrección de estilo. Cualquier error u omisión es responsabilidad íntegra del autor.

[2] Para mayor información revisar UNESCO (2014).

[3] Además de la EBR existe la Educación Básica Especial y la Educación Básica Alternativa. 
[4] El ACP ha sido uno de los métodos más utilizados para la construcción de índices en el ámbito social (salud, educación, entre otros); sin embargo, como cualquier técnica estadística tiene sus limitaciones. Para mayor detalle ver Vences (2014).

[5] Para mayor revisión de los aspectos estadísticos y matemáticos de la construcción del índice ver Mondéjar y Vargas (2008) y Actis di Pasquale (2015).

[6] Se usó la primera componente la cual explicó más del 50\% de la varianza.

\section{BY-NC-ND}

